\title{
Severe exacerbation of Andersen-Tawil syndrome secondary to thyrotoxicosis
}

This article has been corrected since Advance Online Publication, and a corrigendum is also printed in this issue.

\author{
Jordi Díaz-Manera ${ }^{1,2,3}$, Luis Querol ${ }^{1,2,3}$, Aída Alejaldree ${ }^{1,2}$, Ricard Rojas-García ${ }^{1,2}$, Alba Ramos-Fransi ${ }^{1,2}$, \\ Eduard Gallardo ${ }^{1,2}$ and Isabel Illa ${ }^{1,2}$
}

Thyrotoxic periodic paralysis (TPP) is a rare complication of hyperthyroidism characterized by episodes of weakness. Although TPP has been described in patients all over the world, it is especially frequent in Asiatic patients. Recently, two genomewide association studies have found a susceptibility locus on chromosome 17q24.3 near the KCNJ2 gene, which is responsible for another cause of periodic paralysis, the Andersen-Tawil syndrome (ATS). We report the first patient diagnosed with ATS with a de novo c.G899C mutation in the KCNJ2 gene in 2010 who developed an autoimmune hyperthyroidism and TPP in 2013. At the time of the ATS diagnosis other causes of periodic paralysis, including thyroid dysfunction, were ruled out. The condition of the patient, who had mild episodes of proximal weakness at follow-up, deteriorated dramatically in 2013, presenting continuous episodes of severe generalized weakness associated with low levels of potassium requiring frequent admissions to the hospital. After a few months, he also presented signs of hyperthyroidism, and a diagnosis of Grave's disease was made. In our opinion, this case clearly demonstrates that a dysfunction of the Kir2.1 potassium channel encoded by the KCNJ2 gene is a risk factor to develop TPP, and can be a useful tool to identify patients at risk in daily clinics.

Journal of Human Genetics (2014) 59, 465-466; doi:10.1038/jhg.2014.43; published online 22 May 2014

Thyrotoxic periodic paralysis (TPP) is a rare complication of hyperthyroidism characterized by episodes of transient weakness involving proximal muscles of the limbs associated with low potassium levels in serum. ${ }^{1}$ TPP is especially frequent in Asiatic populations but has also been reported in patients with other ethnic origins. Mutations in the KCNJ18 potassium channel gene or the calcium channel $\alpha 1$ subunit gene are associated with TPP susceptibility. ${ }^{2,3}$ Two recent studies have found an association of TPP in Grave's disease patients with a susceptibility locus on chromosome 17q24.3 near the KCNJ2 gene. ${ }^{4,5}$ Mutations in the KCNJ2 gene, which encodes the Kir2.1 potassium channel, are responsible for a dominantly inherited periodic paralysis: the Andersen-Tawil syndrome (ATS). ${ }^{6}$

We describe a 40-year-old Caucasian male diagnosed with ATS who presented a dramatic worsening associated with the development of Graves' disease. In 2010 we visited for the first time a patient with a 30year-long history of infrequent episodes, approximately one every two years, of mild to moderate generalized weakness. The patient had the classical triad of cardiologic anomalies (prominent $\mathrm{U}$ wave in the ECG and episodic bigeminism during sleep), dysmorphic anomalies (lowimplantation ears, hypoplastic mandible, thin upper lip and clinodactilia) and episodes of generalized muscle weakness. We diagnosed ATS with a de novo c.G899C mutation in the KCNJ2 gene. Other causes of periodic paralysis, including thyroid dysfunction, were ruled out at that time (thyroid stimulating hormone (TSH) $1.22 \mathrm{mUIl}^{-1}$; normal range $0.3-5 \mathrm{mUIl}^{-1}$ ). From 2010 to 2013 he had only one episode of weakness that was mild and related to a physical effort. In May 2013 he suddenly developed many episodes of weakness. The episodes were completely different from his previous crises: they were more severe and required admission to the hospital. During these episodes he was unable to lift his arms or legs and had mild dysphagia. Neurological examination showed severe proximal weakness (muscle research council scale (MRC) 2/5 deltoids and biceps and 0/5 psoas and quadriceps) with mild distal muscle involvement. Creatinkinase (CK) levels were normal during the crises, but potassium levels were $<2 \mathrm{mEq}^{-1}$. The patient did not identify any precipitating event. Intravenous potassium administration improved the weakness substantially, but relapses occurred after a few days. Acetazolamide was started, but the severity of the episodes and the hypokalemia increased. Espironolactone was then tried, without any success. Two months later he developed systemic symptoms of hyperthyroidism such as a decrease in body weight, tremor, anxiety, heat intolerance and sweating, but

\footnotetext{
${ }^{1}$ Neurology Department, Hospital de la Santa Creu I Sant Pau de Barcelona, Universitat Autònoma de Barcelona, Barcelona, Spain and ${ }^{2}$ Centro de Investigación Básica en Red en Enfermedades Neurodegenerativas (CIBERNED), Barcelona, Spain

${ }^{3}$ These authors contributed equally to this work.

Correspondence: Dr II Sendra, Neurology Department, Hospital de la Santa Creu I Sant Pau de Barcelona, Universitat Autònoma de Barcelona, C/Sant Antoni Ma Claret 167, 08025 Barcelona, Spain.

E-mail: IIIla@santpau.cat

Received 24 February 2014; revised 30 March 2014; accepted 20 April 2014; published online 22 May 2014
} 
neither goiter nor exophthalmos. Thyroid studies demonstrated high T4 levels (32.9 $\mathrm{pmoll}^{-1}$; normal range 9-19 $\mathrm{pmoll}^{-1}$ ), undetectable thyrotropin levels and antibodies directed against thyrotropin receptor, thyroglobulin and myeloperoxidase. A diagnosis of Graves' disease was established and the patient was treated with methimazol. Normalization of thyroid function was associated with an improvement in the frequency and severity of paralysis episodes and stabilization of plasma potassium levels.

Our interest in this case is based on the fact that thyrotoxicosis produced a profound change in the clinical situation of a patient with a mild ATS. Thyrotoxicosis severely exacerbated the condition of this ATS patient, and it strongly suggests that an underlying dysfunction of the Kir 2.1 channel in the muscle has a role in the development of TPP. In 2012, two different groups identified a TPP susceptibility locus near the KCNJ2 gene in an Asiatic population. ${ }^{4,5}$ They suggested that the muscle expression or function of the Kir 2.1 channel could be involved in a group of TPP patients. However, they did not find any patient who harbored a mutation in the KCNJ2 gene. Hereby, we describe a patient with an already known mutation in the KCNJ2 gene and relatively stable ATS disease that worsened dramatically after developing Graves' disease, strengthening the pathophysiological link between the Kir 2.1 channel and TPP.

TPP is a rare complication of hyperthyroidism in which patients develop episodes of severe weakness that may require hospital admission. ${ }^{1}$ Low potassium levels should be corrected quickly because they increase the risk to suffer lethal arrhythmias. For this reason, it is important to identify patients with a high risk of developing TPP among hyperthyroid patients. Although the pathophysiology of TPP is not completely understood, it has been suggested that hyperactivation of muscle sodium and potassium pumps cause a misbalance in the resting membrane potential. ${ }^{7}$
Screening for mutations on KCNJ2 in TPP might be useful even when the periodic paralysis can be attributed to abnormal thyroid function.

In conclusion, we report the first case of ATS in which a concomitant autoimmune thyrotoxicosis precipitated severe worsening of the underlying disease. Further study into the connection between KCNJ2 gene and TPP susceptibility should include the hypothesis that a dysfunction of the Kir 2.1 channel is a risk factor for TPP in patients with autoimmune hyperthyroidism.

\section{CONFLICT OF INTEREST}

The authors declare no conflict of interest.

1 Hsieh, M. J., Lyu, R. K., Chang, W., Chang, K. H., Chen, C. M., Chang, H. S. et al. Hypokalemic thyrotoxic periodic paralysis: clinical characteristics and predictors of recurrent paralytic attacks. Eur. J. Neurol. 15, 559-564 (2008).

2 Ryan, D. P., da Silva, M. R., Soong, T. W., Fontaine, B., Donaldson, M. R., Kung, A. W. et al. Mutations in potassium channel Kir2.6 cause susceptibility to thyrotoxic hypokalemic periodic paralysis. Cell 140, 88-98 (2010).

3 Kung, A. W., Lau, K. S., Fong, G. C. \& Chan, V. Association of novel single nucleotide polymorphisms in the calcium channel alpha 1 subunit gene $(\mathrm{Ca}(\mathrm{v}) 1.1)$ and thyrotoxic periodic paralysis. J. Clin. Endocrinol. Metab. 89, 1340-1345 (2004).

4 Cheung, C. L., Lau, K. S., Ho, A. Y., Lee, K. K., Tiu, S. C., Lau, E. Y. et al. Genome-wide association study identifies a susceptibility locus for thyrotoxic periodic paralysis at 17q24.3. Nat. Genet. 44, 1026-1029 (2012).

5 Jongiaroenprasert, W. Phusantisampan, T. Mahasirimongkol, S. Mushiroda, T., Hirankarn, N., Snabboon, T. et al. A genome-wide association study identifies novel susceptibility genetic variation for thyrotoxic hypokalemic periodic paralysis. J. Hum. Genet. 57, 301-304 (2012).

6 Díaz-Manera, J., Querol, L., Clarimon, J., Yague, S. \& Illa, I. Unique post-exercise electrophysiological test results in a new Andersen-Tawil syndrome mutation. Clin. Neurophysiol. 122, 2537-2539 (2011)

7 Chan, A., Shinde, R., Chow, C. C., Cockram, C. S. \& Swaminathan, R. In vivo and in vitro sodium pump activity in subjects with thyrotoxic periodic paralysis. BMJ. $\mathbf{3 0 3}$, 1096-1099 (1991) 